\title{
Association Between Levels of Urine Di-(2-ethylhexyl)phthalate Exposure, a Potentially Harmful and Invisible Chemical, and Heart Rate Variability in Young Adults
}

\author{
Ching-Way Chen \\ Jin-Shiang Hwang \\ Academia Sinica \\ Cheng-Chih Hsu \\ National Taiwan University \\ Chien-Yu Lin \\ En Chu Kong Hospital
}

National Taiwan University Hospital Yun Lin Branch

Ta-Chen Su ( $\sim$ tachensu@gmail.com )

National Taiwan University Hospital https://orcid.org/0000-0001-7523-7166

\section{Research}

Keywords: Di-(2-ethylhexyl) phthalate, heart rate variability, young adults

Posted Date: September 27th, 2021

DOI: https://doi.org/10.21203/rs.3.rs-927128/v1

License: (c) (i) This work is licensed under a Creative Commons Attribution 4.0 International License. Read Full License 


\section{Abstract}

\section{Background}

Phthalate exposure is associated with cardiovascular risk. Among the various phthalates, di-(2-ethylhexyl) phthalate (DEHP) is the most important plasticizer in our daily lives. This study investigated the association between DEHP exposure and the alteration of heart rate variability (HRV).

Methods

During 2017-2019, we recruited 974 young adults to investigate the effects of living environments and dietary habits on cardiometabolic disorders in Taiwan. We quantitatively analyzed urinary metabolites of phthalates, including mono-(2-ethylhexyl) phthalate (MEHP), mono-(ethyl-5-hydroxyhexyl) phthalate (MEHHP), and mono-(2-ethyl-5-oxohexyl) phthalate (MEOHP). A continuous electrocardiogram was recorded to obtain a 5-minute ECG. Time-domain and frequency-domain HRV analyses were performed.

Results

Multiple linear regression showed that urinary oxidized DEHP metabolites MEHHP and MEOHP were associated with decreased HRV after controlling for associated cardiovascular risk factors. A higher MEHHP level was associated with a lower TINN (triangular interpolation of NN interval histogram), very-low-frequency (VLF), and low frequency/high frequency (LF/HF) ratio. A higher MEOHP level was associated with a decreased LF/HF ratio. In addition, trend analysis showed that higher MEHHP and MEOHP quantiles were significantly associated with a decreased LF/HF ratio.

Conclusions

DEHP is a potentially harmful and invisible chemicals. The urinary DEHP metabolites MEHHP and MEOHP are associated with decreased HRV, indicating an unfavorable autonomic balance in young adults in Taiwan.

\section{Introduction}

Phthalate esters are colorless and odorless chemicals widely used as plasticizers to add flexibility and resilience to plastic products [1]. Phthalates are essential to make cosmetics, medical devices, plastic, and rubber products. Phthalates are hydrophobic and bound to polymers with noncovalent bonding. Thus, phthalates readily leach into their environment. Phthalates are invisible chemicals hard to detect. Human exposure to phthalates is possible through dietary ingestion, air inhalation, or direct contact. Among various phthalates, di-(2-ethylhexyl) phthalate (DEHP) is the most important plasticizer we encounter in daily life [2].

After entering the human body, DEHP is rapidly degraded to its monoester, mono-(2-ethylhexyl) phthalate (MEHP), which is further metabolized by various hydroxylation and oxidation reactions [3, 4]. Two of the major secondary oxidized DEHP metabolites are mono-(2-ethyl-5-oxohexyl) phthalate (MEOHP) and mono-(2-ethyl-5-hydroxyhexyl) phthalate (MEHHP) [5]. DEHP is eliminated from urine in the form of its metabolites. Although phthalate excretion is rapid with a half-life of less than 24 hours, continuous daily exposure and ingestion of phthalates still cause persistent physiological effects due to steady concentrations in the human body.

Exposure to DEHP is associated with cardiovascular risk factors such as increased blood pressure [6], insulin resistance [7, 8], and diabetes mellitus [9]. Phthalates are also associated with increased inflammation markers of absolute neutrophil counts, alkaline phosphatase, ferritin levels, and C-reactive proteins $[10,11]$. Our previous studies demonstrated that urinary phthalate metabolites were associated with apoptotic microparticles from endothelial cells and platelets, insulin resistance, and subclinical atherosclerosis in terms of increased carotid intima-media thickness $[12,13]$. Urinary phthalates have been associated with a higher risk of stroke in the U.S. [14], and we have also demonstrated that increased DEHP metabolite exposure may be linked to patients with coronary heart diseases [15]. DEHP accelerates atherosclerosis by disturbing cholesterol homeostasis, and the inflammatory response has been ascribed to a pathogenic mechanism in animal models [16]. Our recent study also confirmed that the global DNA methylation marker $5 \mathrm{mdC} / \mathrm{dG}$ may mediate the association between DEHP exposure and subclinical

Page $2 / 13$ 
atherosclerosis [17]. However, these attributable risk factors could not fully explain the underlying adverse effects of DEHP on the cardiac and cardiovascular systems $[15,18]$.

Heart rate variability (HRV) measures the fluctuation of the heart rate. HRV is a noninvasive method to analyze autonomic nervous system effects. For the general population, decreasing heart rate variability is associated with an increased incidence of cardiac events [19] and mortality [20, 21]. Decreased HRV was associated with coronary artery disease [22], heart failure [23], pulmonary hypertension [24], and impaired renal function [25]. For patients with congestive heart failure [26] or after myocardial infarction [27-29], decreased HRV is associated with a higher mortality rate. All this evidence suggests that HRV is a useful tool to evaluate autonomic dysfunction and cardiac adverse effects. In animal models, DEHP-treated mice had decreased HRV, enhanced cardiovascular reactivity, and prolonged blood pressure recovery [30]. This animal model suggested that DEHP may cause adverse cardiac effects through the mechanism of decreasing HRV due to autonomic dysfunction.

However, the association between phthalate exposure and HRV in humans is still unclear. This study was designed to investigate the association between DEHP metabolites and alterations in HRV in young adults with low cardiovascular risk factors.

\section{Subjects And Methods Subjects}

From 1992-2000, a nationwide urine screening program for early renal disease was conducted for Taiwanese children between 6 and 18 years of age [31]. Among over 103,756 students who underwent the screening, 303 children with hypertension and 486 children with normal blood pressure living in the Taipei area joined the YOung TAiwan cohort (YOTA) study between 2006 and 2008 under informed consent and parental agreements in National Taiwan University Hospital [12, 13].

During 2017-2019, we recruited 980 young adults to investigate the effects of living environments and dietary habits on cardiometabolic disorders in Taipei, Taiwan. There were 542 YOTA participants, and 438 young adults of similar age were recruited as the New YOTA cohorts. Among all 980 participants, 4 participants were excluded due to being less than 20 years old or more than 45 years old. Two participants were excluded from the New YOTA study due to no measurable urine sample under hemodialysis status. Thus, 974 participants were enrolled in this study.

\section{Methods}

\section{Assessment of Clinical Information and Risk Stratification}

Basic cardiovascular risk factors, including age, sex, weight, height, diet, smoking habits, alcohol drinking, and exercise habits, and the personal living environment characteristics and dietary habits of each participant were collected. The arterial pressure waveform was recorded by a cuff sphygmomanometer using an oscillometric BP device (DynaPulse 200 M, Pulse Metric Inc., San Diego, CA) [32]. The arterial pressure waveform was measured from left and right hands after at least 5 min of rest in a sitting position in a quiet room. Hypertension was diagnosed if the mean systolic blood pressure was greater than $140 \mathrm{mmHg}$, diastolic pressure was greater than $90 \mathrm{mmHg}$, or the patient was taking anti-hypertension medications.

Blood samples were obtained via the antecubital vein of each participant after overnight fasting for 10-14 h. The serum cholesterol, triglyceride, and low- and high-density lipoprotein cholesterol (LDL-C and HDL-C, respectively) levels and plasma glucose were measured using an autoanalyzer (Toshiba, TBA-200FR; Toshiba, Tokyo, Japan). Biochemical examination for each participant was performed according to standard lab protocols/methods. Diabetes mellitus was diagnosed according to the American Diabetes Association criteria, and those whose fasting glucose levels were equal to or greater than $126 \mathrm{mg} / \mathrm{dL}$ ( 7.0 $\mathrm{mmol} / \mathrm{L}$ ) had diabetes. We measured the weight and height of the participants by standard methods. Body mass index (BMI) was calculated using weight (in kilograms) divided by the square of the height (in meters).

\section{Urinary Phthalates metabolites}

First-voiding morning urine was collected from every participant to analyze urinary metabolites of phthalates, including mono-(2ethylhexyl) phthalate (MEHP), mono-(ethyl-5-hydroxyhexyl) phthalate (MEHHP), and mono-(2-ethyl-5-oxohexyl) phthalate 
(MEOHP). The sample processing for urinary phthalate metabolites was described in detail in our previous reports $[8,13,33]$. Finally, urine mixtures were quantitatively analyzed by liquid chromatography with a tandem mass spectrometric (LC-MS/MS) system.

Regarding the quality assurance and control of DEHP metabolites, we prepared blank samples for each batch of samples during sample preparation. Internal quality control was performed using pooled quality control urine samples, with precision ranging from $6-26 \%$, depending on the metabolite. Alongside pooled urine samples for each batch, low-concentration (20 mg/L) and highconcentration $(50 \mathrm{mg} / \mathrm{L})$ quality control materials were also analyzed. The method detection limits of MEHP, MEHHP, and MEOHP were $0.7,0.1$, and $0.1 \mu \mathrm{g} / \mathrm{L}$, respectively. External quality assurance was assessed using the German External Quality Assessment Scheme for Biological Monitoring (G-EQUAS) [13].

\section{Heart Rate Variability Analysis}

After blood sample collection and a 15-min rest for each participant, we immediately performed the resting electrocardiogram (ECG) examination in the supine position during the daytime (9:00 a.m. to 12:00 p.m.) using an HRV+ (BeneGear, Taipei, Taiwan) with a sampling rate of $250 \mathrm{~Hz}$ (4 ms). A complete 5-min segment of the $\mathrm{N}-\mathrm{N}$ interval was taken for HRV analysis.

Time-domain and frequency-domain analyses were analyzed for heart rate variability. All analyses were performed according to the recommendations of the European Society of Cardiology and the North American Society of Pacing and Electrophysiology[34]. The time-domain measurements of HRV included the mean of the R-R intervals, the standard deviation of the normal-to-normal intervals (SDNN), root mean square successive differences (RMSSDs), between adjacent normal-to-normal intervals and percentage of absolute differences in normal RR intervals greater than $50 \mathrm{~ms}$ (pNN50), and TINN (triangular interpolation of the $\mathrm{NN}$ interval histogram), indicating the baseline width of the RR interval histogram. The frequency-domain measurements of HRV included very-low-frequency (VLF, $<0.04 \mathrm{~Hz}$ ), low frequency (LF: 0.04-0.15 Hz), high frequency (HF: 0.15-0.40 Hz), and LF/HF ratio, which were calculated by Welch's averaged periodogram of the normal-to-normal intervals. These parameters represented the modulation of sympathetic and parasympathetic activity to heart rate variability. The details of translating ECG wave complexes to HRV indices are given in our previous study [35].

\section{Statistical Analysis}

We performed statistical analysis using IBM SPSS Statistics for Windows, Version 26.0. (IBM Corp., Armonk, NY, US). Three urine DEHP metabolites (MEHP, MEHHP, and MEOHP) were log-transformed to fit a normal distribution, as confirmed by the Kolmogorov-Smirnov test. Samples of urine phthalate metabolites below the detection limit were recorded as half of the detection limit. The concentration of urine phthalate metabolites was calibrated by urine creatinine. Phthalate metabolite concentrations are expressed as the mean \pm standard deviation. The Student's t-test was performed to compare the concentrations of urinary phthalate metabolites in different subgroups. The HRV parameters following a nonnormal distribution were also log-transformed. A multiple linear regression model was used to evaluate the dose-response relationships between HRV parameters and urine phthalate metabolites. To adjust the effects of possible confounders, covariates of age, sex, BMI z-score, systolic BP, fasting blood sugar, and LDL-C were added into the multiple linear regression model. The estimated coefficient of urinary phthalate metabolites and its $95 \%$ confidence interval $(95 \% \mathrm{Cl})$ were calculated to measure the effect of 1-unit increase of phthalates metabolites to HRV parameters after adjusting the covariates. We considered an estimate was statistically significant when the $p$ value was less than 0.05. To confirm the association between the concentration of urinary phthalate metabolites and HRV parameters, we divided the participants into four exposure groups according to the quantiles of phthalate metabolite concentrations. Trend analysis in oneway analysis of variance (ANOVA) was performed to test linear trend of HRV parameters among the four exposure groups.

\section{Results}

\section{Participants Characteristics}

Among the 974 participants recruited in this study, there were 407 men and 567 women. The mean age was 31.9 years old. The geometric means and standard deviations of the creatinine-adjusted urinary DEHP metabolites of different characteristic subgroups are listed in Table 1. Women had statistically significantly higher levels of MEHHP and MEOHP than men. Participants 
aged between 18 and 32 had a higher level of MEHP and a lower level of MEOHP. Participants with normal LDL-C had a higher level of MEOHP. Participants with higher education had significantly lower levels of MEHHP and MEOHP. Other clinical characteristics such as hypertension, diabetes mellitus, body mass index, and current smoking had no significant association with urinary DEHP metabolites (Table 1).

Table 1

Basic demographics and subgroup analysis of urinary phthalates metabolites

\begin{tabular}{|c|c|c|c|c|c|c|c|}
\hline & \multicolumn{3}{|c|}{ log MEHP ( $\mu \mathrm{g} / \mathrm{g}$ creatinine) } & \multicolumn{2}{|c|}{ log MEHHP ( $\mu \mathrm{g} / \mathrm{g}$ creatinine) } & \multicolumn{2}{|c|}{$\log$ MEOHP ( $\mu \mathrm{g} / \mathrm{g}$ creatinine) } \\
\hline & $\mathrm{N}(\%)$ & Mean (SD) & $\mathrm{p}$ value & Mean (SD) & $p$ value & Mean (SD) & $p$ value \\
\hline Total & $974(100)$ & $0.682(0.693)$ & & $0.783(0.296)$ & & $-0.386(0.327)$ & \\
\hline Sex & & & 0.193 & & 0.001 & & $<0.001$ \\
\hline Male & $407(41.8)$ & $0.648(0.709)$ & & $0.747(0.297)$ & & $-0.448(0.332)$ & \\
\hline Female & $567(58.2)$ & $0.707(0.681)$ & & $0.809(0.293)$ & & $-0.341(0.316)$ & \\
\hline Age & & & 0.040 & & 0.303 & & 0.048 \\
\hline $18-32$ & $453(46.5)$ & $0.725(0.720)$ & & $0.773(0.281)$ & & $-0.408(0.320)$ & \\
\hline$>32$ & $521(53.5)$ & $0.634(0.659)$ & & $0.792(0.309)$ & & $-0.367(0.332)$ & \\
\hline Hypertension & & & 0.097 & & 0.132 & & 0.314 \\
\hline Yes & $41(4.2)$ & $0.916(0.905)$ & & $0.858(0.317)$ & & $-0.329(0.365)$ & \\
\hline No & $933(95.8)$ & $0.673(0.681)$ & & $0.780(0.295)$ & & $-0.388(0.325)$ & \\
\hline Diabetes mellitus & & & 0.536 & & 0.102 & & 0.290 \\
\hline Yes & $30(3.1)$ & $0.759(0.674)$ & & $0.858(0.245)$ & & $-0.337(0.252)$ & \\
\hline No & $944(96.9)$ & $0.680(0.694)$ & & $0.781(0.298)$ & & $-0.388(0.329)$ & \\
\hline LDL-C, mg/dL & & & 0.532 & & 0.084 & & 0.010 \\
\hline$\geq 130$ & $273(28)$ & $0.660(0.736)$ & & $0.757(0.294)$ & & $-0.429(0.324)$ & \\
\hline$<130$ & $701(72)$ & $0.692(0.676)$ & & $0.794(0.297)$ & & $-0.369(0.124)$ & \\
\hline BMI Z-score & & & 0.740 & & 0.789 & & 0.613 \\
\hline$\geq-0.20$ & $511(52.5)$ & $0.676(0.718)$ & & $0.781(0.309)$ & & $-0.391(0.344)$ & \\
\hline$<-0.20$ & $463(47.5)$ & $0.690(0.665)$ & & $0.786(0.281)$ & & $-0.380(0.307)$ & \\
\hline Smoking habit & & & 0.513 & & 0.270 & & 0.089 \\
\hline Smoker & 177 (18.2) & $0.652(0.682)$ & & $0.761(0.299)$ & & $-0.426(0.345)$ & \\
\hline Nonsmoker & 797 (81.8) & $0.689(0.696)$ & & $0.788(0.295)$ & & $-0.377(0.323)$ & \\
\hline Education & & & 0.469 & & 0.020 & & 0.001 \\
\hline College & $881(90.5)$ & $0.677(0.695)$ & & $0.775(0.288)$ & & $-0.397(0.320)$ & \\
\hline High school & $93(9.5)$ & $0.731(0.677)$ & & $0.864(0.353)$ & & $-0.278(0.370)$ & \\
\hline \multicolumn{8}{|c|}{ Abbreviations: LDL-C: low-density lipoprotein cholesterol, and BMI: body mass index } \\
\hline $\mathrm{p}$ value determine & y t-test & & & & & & \\
\hline
\end{tabular}

\section{Heart Rate Variability Analysis}


Univariable analysis of the time domain showed that higher levels of MEHHP and MEOHP were associated with decreased TINN (Table 2). Frequency domain analysis revealed that higher levels of MEHHP and MEOHP were associated with a lower VLF, LF, and LF/HF ratio, as shown in Table 3.

Table 2

Univariable linear regression of urinary phthalate metabolites and time-domain heart rate variability (HRV) analysis parameters

\begin{tabular}{|c|c|c|c|c|c|c|c|c|c|c|c|c|}
\hline \multirow[t]{2}{*}{ HRV } & \multicolumn{2}{|c|}{ log Mean RRI } & \multicolumn{2}{|c|}{$\log$ SDNN (ms) } & \multicolumn{2}{|c|}{$\begin{array}{l}\log \text { RMSSD } \\
(\mathrm{ms})\end{array}$} & \multicolumn{2}{|c|}{ log pNN50 (\%) } & \multicolumn{2}{|c|}{$\begin{array}{l}\text { log HRV } \\
\text { triangular index }\end{array}$} & \multicolumn{2}{|c|}{$\log$ TINN (ms) } \\
\hline & $\begin{array}{l}\text { Beta } \\
\text { (SE) }\end{array}$ & $\mathrm{p}$ & $\begin{array}{l}\text { Beta } \\
\text { (SE) }\end{array}$ & $\mathrm{p}$ & $\begin{array}{l}\text { Beta } \\
\text { (SE) }\end{array}$ & $\mathrm{p}$ & $\begin{array}{l}\text { Beta } \\
\text { (SE) }\end{array}$ & $\mathrm{p}$ & $\begin{array}{l}\text { Beta } \\
\text { (SE) }\end{array}$ & $\mathrm{p}$ & $\begin{array}{l}\text { Beta } \\
\text { (SE) }\end{array}$ & $\mathrm{p}$ \\
\hline MEHP & $\begin{array}{l}0.001 \\
(0.009)\end{array}$ & 0.786 & $\begin{array}{l}-0.012 \\
(0.009)\end{array}$ & 0.182 & $\begin{array}{l}-0.005 \\
(0.012)\end{array}$ & 0.645 & $\begin{array}{l}-0.021 \\
(0.032)\end{array}$ & 0.507 & $\begin{array}{l}-0.008 \\
(0.008)\end{array}$ & 0.299 & $\begin{array}{l}-0.011 \\
(0.010)\end{array}$ & 0.266 \\
\hline MEHHP & $\begin{array}{l}0.006 \\
(0.007)\end{array}$ & 0.349 & $\begin{array}{l}-0.032 \\
(0.020)\end{array}$ & 0.121 & $\begin{array}{l}-0.003 \\
(0.028)\end{array}$ & 0.903 & $\begin{array}{l}0.039 \\
(0.074)\end{array}$ & 0.597 & $\begin{array}{l}-0.041 \\
(0.018)\end{array}$ & 0.025 & $\begin{array}{l}-0.066 \\
(0.023)\end{array}$ & 0.005 \\
\hline MEOHP & $\begin{array}{l}0.004 \\
(0.006)\end{array}$ & 0.495 & $\begin{array}{l}-0.034 \\
(0.019)\end{array}$ & 0.071 & $\begin{array}{l}-0.008 \\
(0.026)\end{array}$ & 0.751 & $\begin{array}{l}0.035 \\
(0.068)\end{array}$ & 0.608 & $\begin{array}{l}-0.040 \\
(0.017)\end{array}$ & 0.016 & $\begin{array}{l}-0.051 \\
(0.021)\end{array}$ & 0.017 \\
\hline
\end{tabular}

MEHP, MEHHP and MEOHP: log transformations with creatinine-adjusted ( $\mu \mathrm{g} / \mathrm{g}$ creatinine)

Abbreviations: MEHP: Mono-(2-ethylhexyl) phthalate, MEHHP: mono (2-ethyl-5-hydroxyhexyl) phthalate, MEOHP: mono-(2ethyl-5-oxohexyl) phthalate, RRI: R-R intervals, SDNN: Standard deviation of NN intervals, RMSSD: Root mean square of successive RR interval differences, pNN50: Percentage of successive RR intervals that differ by more than 50 ms, and TINN: Baseline width of the RR interval histogram, Beta: beta coefficient, and SE: standard error

Table 3

Univariable linear regression of urinary phthalate metabolites and frequency-domain heart rate variability (HRV) analysis parameters

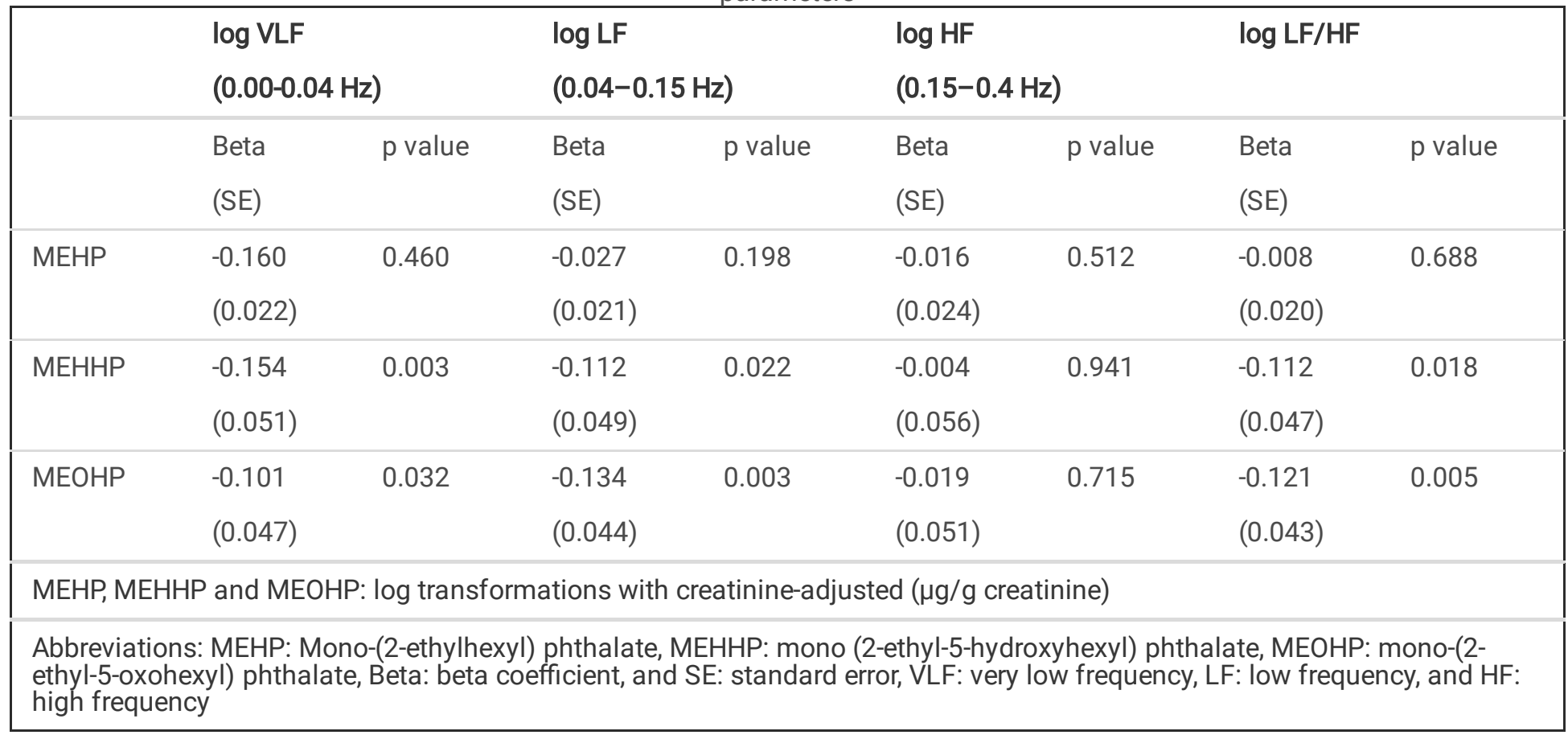

Univariable analysis of HRV showed that older age; being a woman; higher BMI z-score; and higher BP, blood sugar, and LDL-C were negatively associated with time-domain HRV in the lower mean RR interval, SDNN, RMSSD, pNN50, and TINN. In addition, we also showed a negative association between cardiovascular risk factors and frequency domain HRV for the VLF, LF, HF, and the LF/HF ratio (Supplemental Table 1 and Supplemental Table 2). 


\section{Multiple Linear Regression Models}

In the multiple linear regression analysis of the HRV parameters after adjusting for age, sex, BMI z-score, blood pressure, fasting blood glucose, and LDL-C, we demonstrated that a higher MEHHP level was associated with a significantly lower TINN, with an estimated coefficient of -0.057 and $95 \% \mathrm{Cl}=(-0.102 \sim-0.011)$, VLF with $-0.153(-0.210 \sim-0.010)$, and LF/HF ratio -0.110 $(-0.200 \sim-0.020)$ (Table 4). A higher MEOHP level was associated with a significant decrease of LF/HF ratio with an estimated coefficient of $-0.101(-0.184 \sim-0.019)$ (Table 5).

Table 4

Multivariable linear regression of urinary phthalate metabolites and time-domain HRV analysis parameters

\begin{tabular}{|c|c|c|c|c|}
\hline & \multicolumn{2}{|l|}{ log HRV triangular index } & \multicolumn{2}{|l|}{$\log$ TINN (ms) } \\
\hline & Beta $(95 \% \mathrm{Cl})$ & $\mathrm{p}$ value & Beta $(95 \% \mathrm{Cl})$ & $\mathrm{p}$ value \\
\hline MEHP & $-0.001(-0.015 \sim 0.014)$ & 0.918 & $-0.008(-0.270 \sim 0.012)$ & 0.432 \\
\hline MEHHP & $-0.019(-0.053 \sim 0.016)$ & 0.290 & $-0.057(-0.102 \sim-0.011)$ & 0.016 \\
\hline MEOHP & $-0.017(-0.048 \sim 0.015)$ & 0.310 & $-0.040(-0.082 \sim 0.003)$ & 0.067 \\
\hline \multicolumn{5}{|c|}{ Adjusted Model: linear regression adjusted for age, sex, BMI z-score, systolic blood pressure, fasting sugar and LDL-C } \\
\hline \multicolumn{5}{|c|}{ MEHP, MEHHP and MEOHP: log transformations with creatinine-adjusted ( $\mu \mathrm{g} / \mathrm{g}$ creatinine) } \\
\hline
\end{tabular}

Table 5

Multivariable linear regression of urinary phthalate metabolites and frequency-domain HRV analysis parameters

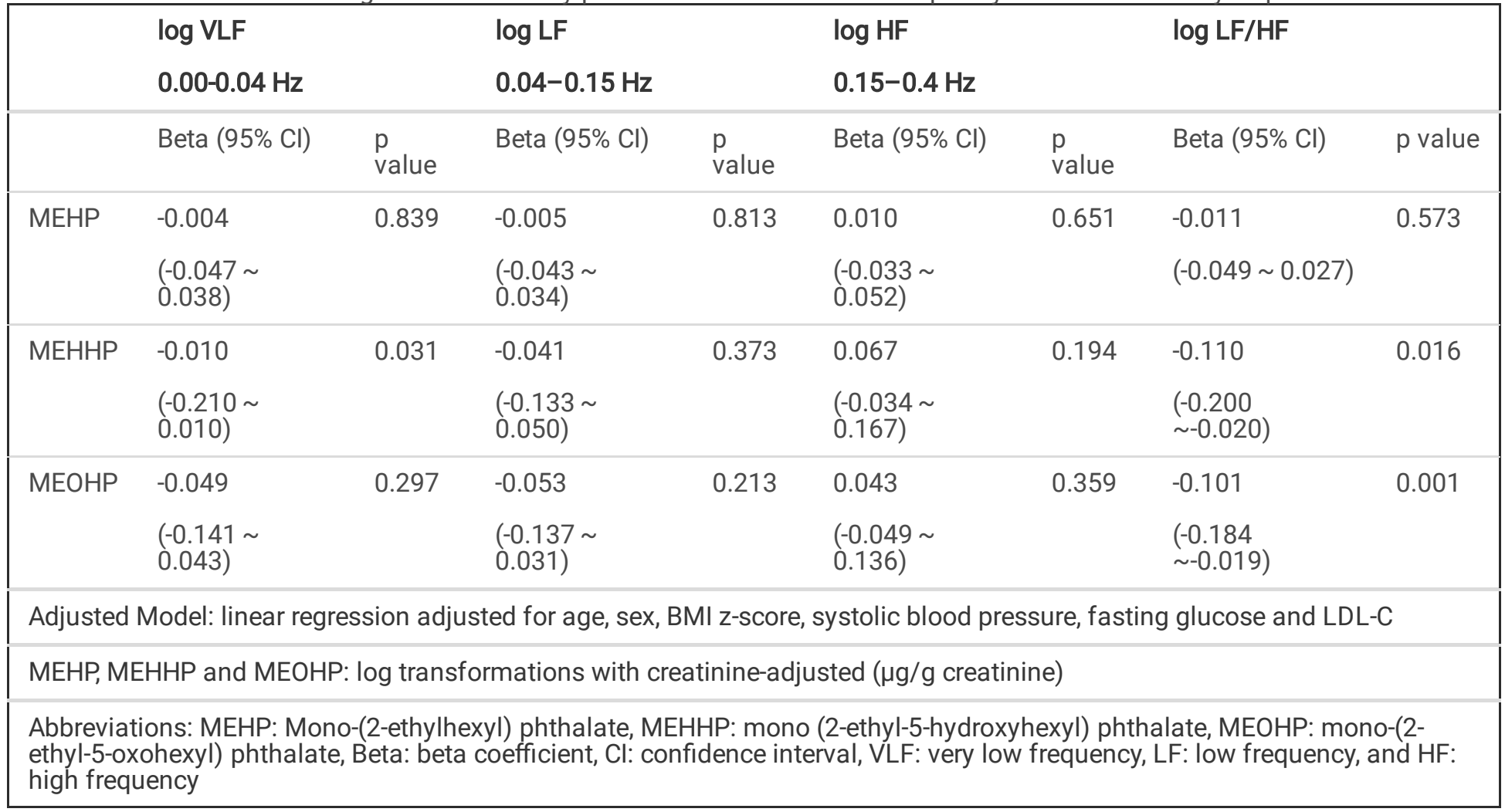

The trend analysis in one-way ANOVA showed that exposure to higher MEHHP and MEOHP quantiles were significantly associated with a lower LF/HF ratio. The $p$ values for trend were 0.014 for MEHHP and 0.001 for MEOHP (Table 6 and Fig. 1).

Table 6 One-way analysis of variance for urinary phthalate metabolites and the autonomic balance, LF/HF ratio 
$\log \mathrm{LF} / \mathrm{HF}$

\begin{tabular}{|c|c|c|c|c|c|c|c|}
\hline log MEHHP & Mean & SD & $\mathrm{p}$-value for linear trend & log MEOHP & Mean & SD & $\mathrm{p}$-value for linear trend \\
\hline \multirow[t]{2}{*}{ 1st quartile (<0.589) } & 0.137 & 0.446 & 0.014 & 1st quartile & 0.143 & 0.450 & 0.001 \\
\hline & & & & $(<-0.592)$ & & & \\
\hline \multirow[t]{2}{*}{ 2nd quartile $(<0.774)$} & 0.112 & 0.386 & & 2nd quartile & 0.123 & 0.411 & \\
\hline & & & & $(<-0.395)$ & & & \\
\hline \multirow[t]{2}{*}{ 3rd quartile (<0.965) } & 0.054 & 0.482 & & 3rd quartile & 0.084 & 0.437 & \\
\hline & & & & $(<-0.185)$ & & & \\
\hline 4th quartile & 0.088 & 0.438 & & 4th quartile & 0.089 & 0.452 & \\
\hline$\left({ }^{3} 0.965\right)$ & & & & $\left({ }^{3}-0.185\right)$ & & & \\
\hline
\end{tabular}

Log MEHHP and Log MEOHP: log transformations with creatinine-adjusted ( $\mu \mathrm{g} / \mathrm{g}$ creatinine)

Abbreviations: MEHHP: mono (2-ethyl-5-hydroxyhexyl) phthalate, MEOHP: mono-(2-ethyl-5-oxohexyl) phthalate, LF: low-frequency, HF: high frequency

$p$ for testing trend of means by one-way analysis of variance (ANOVA) mo

\section{Discussion}

The most important finding of this study is that urinary phthalate metabolites were associated with decreased HRV in young adults. This is the first report to show an association between DEHP exposure and decreased autonomic balance in a human study.

Previous studies have shown that phthalate DEHP exposure alters the autonomic nervous system and decreases HRV in animal models [30]. DEHP exposure also increased the expression of the genes encoding endothelin-1 and angiotensin-converting enzyme in heart tissue [30]. Other studies showed that DEHP-treated cardiomyocytes had increased gene expression of calcium handling genes [36] and subsequently markedly reduced cardiac network synchronicity of DEHP-treated cardiomyocytes [37]. These were possible mechanisms of decreased HRV caused by DEHP exposure.

Endothelial function was associated with heart rate variability in animal studies [38]. Endothelial dysfunction was also associated with decreased HRV in healthy adults and patients with stable coronary artery disease or diabetes mellitus [39-41]. Increased carotid intima-media thickness was associated with decreased HRV in previous studies [42, 43]. Our previous studies showed that urinary phthalate metabolites were associated with endothelial dysfunction, insulin resistance and increased carotid intima-media thickness $[12,13,44]$. Thus, exposure to DEHP may mediate impaired HRV through these mechanisms.

Our study showed that higher concentrations of urinary MEHHP and MEOHP were associated with decreased HRV, primarily in frequency domain HRV. We did not identify this association in MEHP, which is the monoester of DEHP after the first step of hydrolysis metabolism. Both MEHHP and MEOHP were two of the major secondary and oxidized urinary metabolites of DEHP. The possible explanations for the results of our study are as follows. First, the formation of MEHP from DEHP is possible through abiotic hydrolysis during urinary sample collection, storage, and processing. MEHHP and MEOHP are secondary oxidized DEHP metabolites in the liver and thus are less likely to be contaminated during sampling handling [5]. Second, urinary MEHP accounts for less than $10 \%$ of DEHP intake [45]. Urinary MEHP also has the shortest half-life (approximately 5 hours) compared to urinary MEHHP or MEOHP (both approximately 10 hours) [4]. Urinary MEHP could thus be more easily influenced by prolonged sample handling during urine collection at the study site. For example, it took approximately 4-5 hours before urine was sent to the refrigerator to freeze in this study. Thus, urinary MEHP might not be able to reflect the actual DEHP exposure and its pathophysiological effects on autonomic dysfunction.

Page $8 / 13$ 
HRV analysis includes time-domain analysis, frequency-domain analysis, and nonlinear regression. We used time-domain and frequency-domain HRV analysis in our study as studies showed that linear and nonlinear HRV analyses yielded similar conclusions [46, 47]. Multivariable linear regression was performed to control for the possible confounding factors such as age, sex, BMI Z-score, SBP, fasting glucose, and LDL. Thus, the association between decreased HRV and urinary DEHP oxidized metabolites was less likely due to other confounding factors.

This study has many strengths. First, most cardiovascular risk factors such as older age; a higher BMI z-score; and higher BP, blood sugar, and LDL-C were mostly associated with lower time-domain and frequency-domain HRV, which also corroborated the reliable measurement of HRV. Second, measurements of phthalate metabolites are compatible with our previous study in which women had higher levels of DEHP metabolites than men [13]. Third, even after controlling for most associated confounders, DEHP metabolites were still strongly linked to reduced HRV parameters. Fourth, decreased HRV was noted in various physiological and pathophysiological statuses, such as major cardiovascular risk factors. This evidence also corroborates that the validity of HRV measurements. We recruited young adults with low cardiovascular risk and prevented these possible confounding factors.

This study also has several limitations. First, this was a cross-sectional study as we initiated a new YOung TAiwan cohort (YOTA) study. A previous study showed a temporal trend of decreased urinary phthalate concentrations 5 years after the regulation of phthalates [48]. Even though this is the first study reporting decreased HRV associated with DEHP exposure, a future follow-up is warranted to confirm the temporal trend and causality of HRV alterations due to phthalate exposure. Second, a single measurement of first-morning spot urine may not represent the real phthalate exposure of each participant. We could not confirm that this single measurement could represent the steady-state of the daily phthalate exposure of each individual. Repeated measurement studies for measurement reliability and consistency are needed in the future.

\section{Conclusion}

DEHP is a potentially harmful and invisible chemical. Urinary DEHP oxidized metabolites MEHHP and MEOHP are associated with decreased HRV in time-domain and frequent-domain analysis. Preventing phthalate exposure, particularly DEHP, could prevent environmental pollution and minimize its cardiovascular hazards to humans. A prospective cohort study is warranted to confirm the causality of DEHP exposure and decreased HRV.

\section{Declarations}

\section{ACKNOWLEDGMENTS}

We thank all participants who were recruited in the National Health Research Institute grant-supported study during 2017-2019 (NHRI-EX106-10629PI), with the grant proposal "Effects of living environments and dietary habits on cardiometabolic disorders in young adults". We thank associate professor Cheng-Chih Hsu for the measurements of urinary phthalate metabolites by the Mass Spectrometry, Analytical Chemistry Laboratory. We thank Dr. Kuo-Tong Huang and Nan-Chang Clinical Laboratory for the study space and laboratory analysis support. We also thank the 3rd core laboratory of National Taiwan University Hospital.

\section{AUTHOR CONTRIBUTION STATEMENTS}

Ching-Way Chen: data management and analysis, data interpretation and manuscript drafting

Jin-Shiang Hwang: statistical analysis and manuscript critical review and comments

Cheng-Chih Hsu, urinary phthalate metabolite measurement

Chien-Yu Lin: manuscript critical review and comments

Ta-Chen Su: designed and conducted the study, subject recruitment, data management and analysis, data interpretation, manuscript draft and critical review, manuscript comments and revisions 
There was no conflict of interest to declare.

The study was approved by the Institutional Review Boards at National Taiwan University Hospital,

Taiwan (IRB No: 201604089RINA). Informed consent was obtained from all participants.

\section{SOURCE of FUNDING}

This study was sponsored by grants from the National Health Research Institute of

Taiwan (NHRI EX95-9531PI and NHRI EX106-10629PI).

\section{DISCLOSURES}

The authors have nothing to disclose.

\section{References}

1. Kamrin MA: Phthalate risks, phthalate regulation, and public health: a review. J Toxicol Environ Health B Crit Rev 2009, 12(2):157-174.

2. Lorz PM, Rowae FK, Enke W, Jäckh R, Bhargava N, Hillesheim W (eds.): Phthalic acid and derivatives, 7th edn. Weinheim, Germany: Wiley-VCH; 2007.

3. Koch HM, Bolt HM, Angerer J: Di(2-ethylhexyl)phthalate (DEHP) metabolites in human urine and serum after a single oral dose of deuterium-labelled DEHP. Archives of Toxicology 2004, 78(3):123-130.

4. Koch HM, Preuss R, Angerer J: Di(2-ethylhexyl)phthalate (DEHP): human metabolism and internal exposure - an update and latest results1. International Journal of Andrology 2006, 29(1):155-165.

5. Kato K, Silva MJ, Reidy JA, Hurtz D, 3rd, Malek NA, Needham LL, Nakazawa H, Barr DB, Calafat AM: Mono(2-ethyl-5hydroxyhexyl) phthalate and mono-(2-ethyl-5-oxohexyl) phthalate as biomarkers for human exposure assessment to di-(2ethylhexyl) phthalate. Environ Health Perspect 2004, 112(3):327-330.

6. Trasande L, Sathyanarayana S, Spanier AJ, Trachtman H, Attina TM, Urbina EM: Urinary phthalates are associated with higher blood pressure in childhood. J Pediatr 2013, 163(3):747-753.e741.

7. Trasande L, Spanier AJ, Sathyanarayana S, Attina TM, Blustein J: Urinary phthalates and increased insulin resistance in adolescents. Pediatrics 2013, 132(3):e646-655.

8. Chen SY, Hwang JS, Sung FC, Lin CY, Hsieh CJ, Chen PC, Su TC: Mono-2-ethylhexyl phthalate associated with insulin resistance and lower testosterone levels in a young population. Environ Pollut 2017, 225:112-117.

9. James-Todd T, Stahlhut R, Meeker JD, Powell SG, Hauser R, Huang T, Rich-Edwards J: Urinary phthalate metabolite concentrations and diabetes among women in the National Health and Nutrition Examination Survey (NHANES) 2001-2008. Environ Health Perspect 2012, 120(9):1307-1313.

10. Ferguson KK, Loch-Caruso R, Meeker JD: Exploration of oxidative stress and inflammatory markers in relation to urinary phthalate metabolites: NHANES 1999-2006. Environ Sci Technol 2012, 46(1):477-485.

11. Ferguson KK, Loch-Caruso R, Meeker JD: Urinary phthalate metabolites in relation to biomarkers of inflammation and oxidative stress: NHANES 1999-2006. Environ Res 2011, 111(5):718-726.

12. Lin CY, Hsieh CJ, Lo SC, Chen PC, Torng PL, Hu A, Sung FC, Su TC: Positive association between concentration of phthalate metabolites in urine and microparticles in adolescents and young adults. Environ Int 2016, 92-93:157-164. 
13. Su TC, Hwang JS, Torng PL, Wu C, Lin CY, Sung FC: Phthalate exposure increases subclinical atherosclerosis in young population. Environ Pollut 2019, 250:586-593.

14. Shiue I: Urine phthalate concentrations are higher in people with stroke: United States National Health and Nutrition Examination Surveys (NHANES), 2001-2004. Eur J Neurol 2013, 20(4):728-731.

15. Su TC, Hwang JJ, Sun CW, Wang SL: Urinary phthalate metabolites, coronary heart disease, and atherothrombotic markers. Ecotoxicol Environ Saf 2019, 173:37-44.

16. Zhao JF, Hsiao SH, Hsu MH, Pao KC, Kou YR, Shyue SK, Lee TS: Di-(2-ethylhexyl) phthalate accelerates atherosclerosis in apolipoprotein E-deficient mice. Arch Toxicol 2016, 90(1):181-190.

17. Lin CY, Lee HL, Hwang YT, Wang C, Hsieh CJ, Wu C, Sung FC, Su TC: The association between urine di-(2-ethylhexyl) phthalate metabolites, global DNA methylation, and subclinical atherosclerosis in a young Taiwanese population. Environ Pollut 2020, 265(Pt B):114912.

18. Posnack NG: The adverse cardiac effects of Di(2-ethylhexyl)phthalate and Bisphenol A. Cardiovasc Toxicol 2014, 14(4):339-357.

19. Tsuji H, Larson MG, Venditti FJ, Jr., Manders ES, Evans JC, Feldman CL, Levy D: Impact of reduced heart rate variability on risk for cardiac events. The Framingham Heart Study. Circulation 1996, 94(11):2850-2855.

20. Dekker JM, Crow RS, Folsom AR, Hannan PJ, Liao D, Swenne CA, Schouten EG: Low heart rate variability in a 2-minute rhythm strip predicts risk of coronary heart disease and mortality from several causes: the ARIC Study. Atherosclerosis Risk In Communities. Circulation 2000, 102(11):1239-1244.

21. Hillebrand S, Gast KB, de Mutsert R, Swenne CA, Jukema JW, Middeldorp S, Rosendaal FR, Dekkers OM: Heart rate variability and first cardiovascular event in populations without known cardiovascular disease: meta-analysis and dose-response meta-regression. Europace 2013, 15(5):742-749.

22. Kotecha D, New G, Flather MD, Eccleston D, Pepper J, Krum H: Five-minute heart rate variability can predict obstructive angiographic coronary disease. Heart 2012, 98(5):395-401.

23. Tsai CH, Ma HP, Lin YT, Hung CS, Huang SH, Chuang BL, Lin C, Lo MT, Peng CK, Lin YH: Usefulness of heart rhythm complexity in heart failure detection and diagnosis. Sci Rep 2020, 10(1):14916.

24. Tsai CH, Ma HP, Lin YT, Hung CS, Hsieh MC, Chang TY, Kuo PH, Lin C, Lo MT, Hsu HH et al: Heart Rhythm Complexity Impairment in Patients with Pulmonary Hypertension. Sci Rep 2019, 9(1):10710.

25. Chen J, Zhou X, Zhang H, Liu Y, Cao C, Dong R, Yuan Y, Wang M, Lu Y, Wu M et al: Association between urinary concentration of phthalate metabolites and impaired renal function in Shanghai adults. Environmental Pollution 2019, 245:149162.

26. Nolan J, Batin PD, Andrews R, Lindsay SJ, Brooksby P, Mullen M, Baig W, Flapan AD, Cowley A, Prescott RJ et al: Prospective study of heart rate variability and mortality in chronic heart failure: results of the United Kingdom heart failure evaluation and assessment of risk trial (UK-heart). Circulation 1998, 98(15):1510-1516.

27. Kleiger RE, Miller JP, Bigger JT, Jr., Moss AJ: Decreased heart rate variability and its association with increased mortality after acute myocardial infarction. Am J Cardiol 1987, 59(4):256-262.

28. Bigger JT, Jr., Fleiss JL, Steinman RC, Rolnitzky LM, Kleiger RE, Rottman JN: Frequency domain measures of heart period variability and mortality after myocardial infarction. Circulation 1992, 85(1):164-171.

29. Buccelletti E, Gilardi E, Scaini E, Galiuto L, Persiani R, Biondi A, Basile F, Silveri NG: Heart rate variability and myocardial infarction: systematic literature review and metanalysis. Eur Rev Med Pharmacol Sci 2009, 13(4):299-307. 
30. Jaimes R, 3rd, Swiercz A, Sherman M, Muselimyan N, Marvar PJ, Posnack NG: Plastics and cardiovascular health: phthalates may disrupt heart rate variability and cardiovascular reactivity. Am J Physiol Heart Circ Physiol 2017, 313(5):H1044h1053.

31. Su TC, Liao CC, Chien KL, Hsu SH, Sung FC: An overweight or obese status in childhood predicts subclinical atherosclerosis and prehypertension/hypertension in young adults. J Atheroscler Thromb 2014, 21(11):1170-1182.

32. Brinton TJ, Cotter B, Kailasam MT, Brown DL, Chio SS, O'Connor DT, DeMaria AN: Development and validation of a noninvasive method to determine arterial pressure and vascular compliance. Am J Cardiol 1997, 80(3):323-330.

33. Koch HM, Bolt HM, Angerer J: Di(2-ethylhexyl)phthalate (DEHP) metabolites in human urine and serum after a single oral dose of deuterium-labelled DEHP. Arch Toxicol 2004, 78(3):123-130.

34. Heart rate variability: standards of measurement, physiological interpretation and clinical use. Task Force of the European Society of Cardiology and the North American Society of Pacing and Electrophysiology. Circulation 1996, 93(5):1043-1065.

35. Chen SY, Chan CC, Su TC: Particulate and gaseous pollutants on inflammation, thrombosis, and autonomic imbalance in subjects at risk for cardiovascular disease. Environ Pollut 2017, 223:403-408.

36. Posnack NG, Lee NH, Brown R, Sarvazyan N: Gene expression profiling of DEHP-treated cardiomyocytes reveals potential causes of phthalate arrhythmogenicity. Toxicology 2011, 279(1-3):54-64.

37. Gillum N, Karabekian Z, Swift LM, Brown RP, Kay MW, Sarvazyan N: Clinically relevant concentrations of di (2-ethylhexyl) phthalate (DEHP) uncouple cardiac syncytium. Toxicol Appl Pharmacol 2009, 236(1):25-38.

38. Custodis F, Baumhäkel M, Schlimmer N, List F, Gensch C, Böhm M, Laufs U: Heart rate reduction by ivabradine reduces oxidative stress, improves endothelial function, and prevents atherosclerosis in apolipoprotein E-deficient mice. Circulation 2008, 117(18):2377-2387.

39. Fox BM, Brantley L, White C, Seigler N, Harris RA: Association beween resting heart rate, shear and flow-mediated dilation in healthy adults. Exp Physiol 2014, 99(10):1439-1448.

40. Aydin Sunbul E, Sunbul M, Gulec H: The impact of major depression on heart rate variability and endothelial dysfunction in patients with stable coronary artery disease. Gen Hosp Psychiatry 2017, 44:4-9.

41. Bhati P, Alam R, Moiz JA, Hussain ME: Subclinical inflammation and endothelial dysfunction are linked to cardiac autonomic neuropathy in type 2 diabetes. J Diabetes Metab Disord 2019, 18(2):419-428.

42. Hoshi RA, Santos IS, Dantas EM, Andreão RV, Mill JG, Goulart AC, Lotufo PA, Bensenor I: Relationship between heart rate variability and carotid intima-media thickness in the Brazilian Longitudinal Study of Adult Health - ELSA-Brasil. Clin Physiol Funct Imaging 2020, 40(2):122-130.

43. Pereira VL, Jr., Dobre M, Dos Santos SG, Fuzatti JS, Oliveira CR, Campos LA, Brateanu A, Baltatu OC: Association between Carotid Intima Media Thickness and Heart Rate Variability in Adults at Increased Cardiovascular Risk. Front Physio/ 2017, 8:248.

44. Chu PC, Wu C, Su TC: Association between Urinary Phthalate Metabolites and Markers of Endothelial Dysfunction in Adolescents and Young Adults. Toxics 2021, 9(2).

45. Koch HM, Bolt HM, Preuss R, Angerer J: New metabolites of di(2-ethylhexyl)phthalate (DEHP) in human urine and serum after single oral doses of deuterium-labelled DEHP. Archives of Toxicology 2005, 79(7):367-376.

46. Mäkikallio TH, Huikuri HV, Hintze U, Videbaek J, Mitrani RD, Castellanos A, Myerburg RJ, Møller M: Fractal analysis and time-and frequency-domain measures of heart rate variability as predictors of mortality in patients with heart failure. $A m \mathrm{~J}$ Cardiol 2001, 87(2):178-182. 
47. Jovic A, Bogunovic N: Electrocardiogram analysis using a combination of statistical, geometric, and nonlinear heart rate variability features. Artificial Intelligence in Medicine 2011, 51(3):175-186.

48. Tranfo G, Caporossi L, Pigini D, Capanna S, Papaleo B, Paci E: Temporal Trends of Urinary Phthalate Concentrations in Two Populations: Effects of REACH Authorization after Five Years. Int J Environ Res Public Health 2018, 15(9).

\section{Figures}

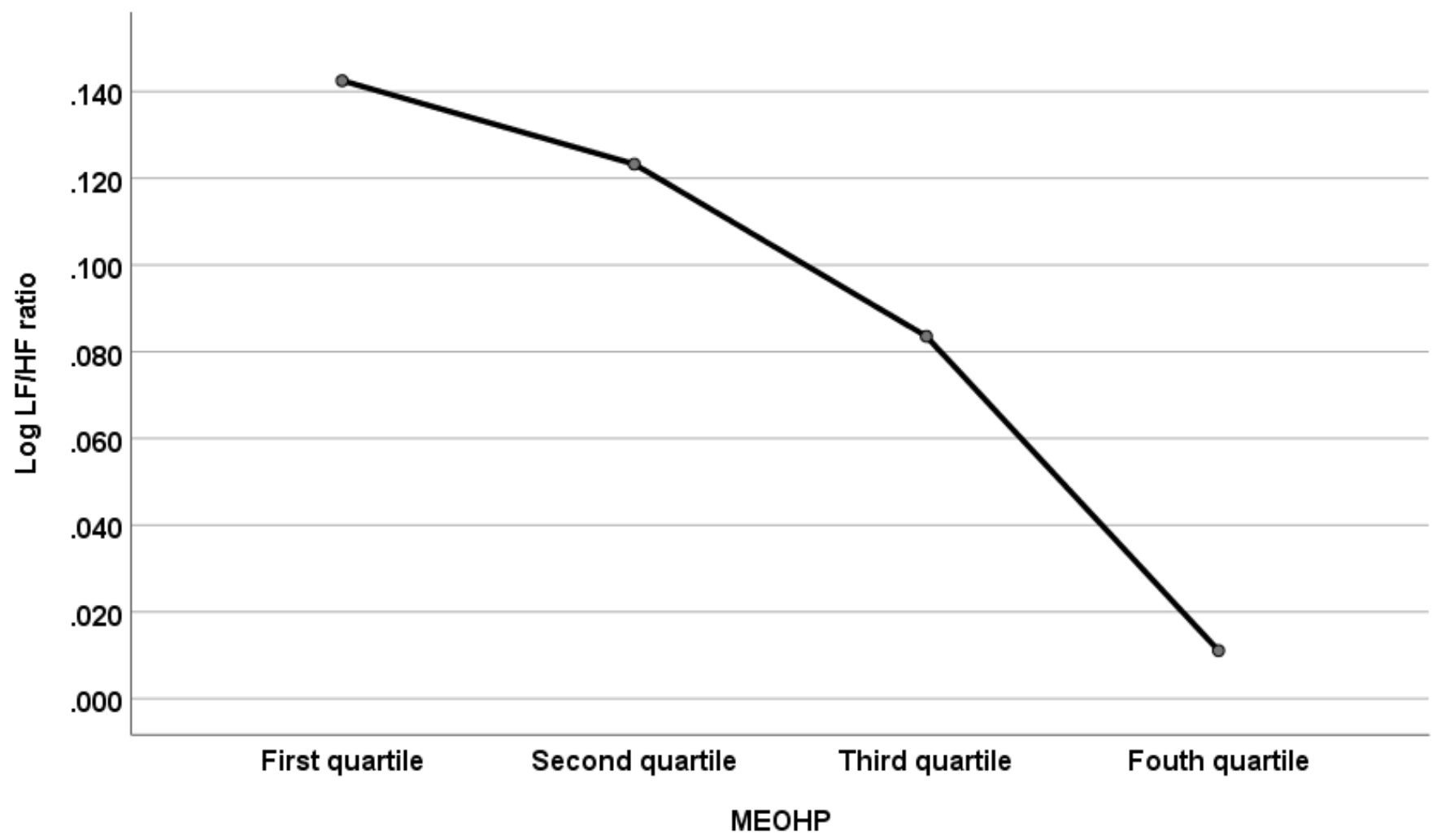

\section{Figure 1}

Means plot of log LF/HF ratio among four exposure groups categorized by the four quartiles of MEOHP measurements. Abbreviations: MEOHP: mono-(2-ethyl-5-oxohexyl) phthalate, LF: low-frequency, HF: high frequency 